\title{
Umgang mit Sterben und Tod
}

\section{Christian Kind}

Prof. Dr. med., Vorsitzender der Subkommission "Umgang mit Sterben und Tod»

\author{
Die Behandlung, Betreuung und Begleitung von Patientinnen und Patienten, die \\ mit dem eigenen Sterben konfrontiert sind, ist eine zentrale Aufgabe der Medizin, \\ die hohen Respekt vor der Würde und dem selbstbestimmten Willen der Patientin \\ und grosse ethische Verantwortung verlangt. Die SAMW hat medizin-ethische \\ Richtlinien ausgearbeitet, die den Umgang mit Sterben und Tod behandeln. \\ Die definitive Fassung der Richtlinien wurde am 17. Mai 2018 vom SAMW-Senat \\ verabschiedet.
}

Wie stark das Thema «Umgang mit Sterben und Tod» bewegt, hat das grosse Echo während der öffentlichen Vernehmlassung gezeigt: Insgesamt sind 118 Stellungnahmen eingetroffen, die meisten sehr umfangreich und differenziert. 92 stammen von Fachgesellschaften, Ethikkommissionen, Verbänden, Institutionen und Behörden und 26 von Einzelpersonen.

Die Mehrheit begrüsste die Erweiterungen gegenüber der Vorgängerrichtlinie «Betreuung von Patientinnen und Patienten am Lebensende», darunter den umfassenderen Geltungsbereich, die Betonung der Wichtigkeit des Gesprächs und die vermehrte Berücksichtigung der Angehörigen. Auch die differenzierte Behandlung der palliativen Sedierung wurde häufig positiv erwähnt.

Erwartungsgemäss stiessen die Bestimmungen zur Suizidhilfe auf die grösste Kritik. Den Vorschlag der SAMW zurückgewiesen haben jedoch nur 19 (22\%), oft waren dies Einzelpersonen. Für 13 dieser ablehnenden Stimmen geht die Öffnung zu weit, vier forderten eine weitergehende Öffnung.

Gestützt auf diese Vernehmlassungsergebnisse haben die Gremien der SAMW beschlossen, den ursprünglichen Vorschlag der Subkommission weitgehend bestehen zu lassen und nur in kleineren Punkten zu modifizieren. Auf mehrfachen Wunsch enthalten die Richtlinien neu eine explizite ethische Begründung für die gewählte Regelung zur Suizidhilfe.

Neben den Änderungen im Kapitel Suizidhilfe wurden gegenüber dem Vernehmlassungstext verschiedene Klärungen und Verbesserungen vorgenommen. Wichtigste Klärung ist die Verdeutlichung der Anwendbarkeit der Richtlinien auch für Kinder jeglichen Alters und Menschen mit geistiger und Mehrfachbehinderung sowie, soweit zeitlich möglich, auch in Notfallsituationen. Zudem wurde ein sprachliches Anliegen aufgenommen: die zuvor kontinuierlich abwechselnden Geschlechtsbezeichnungen werden neu abschnittsweise alternierend verwendet.

\section{Aufbau und Inhalt der Richtlinien}

In der Präambel werden Hintergrund und Zielsetzung der Richtlinien erläutert. Diese sollen die betreuenden Fachpersonen unterstützen bei einem von den Prinzipien der Palliative Care geleiteten Umgang mit Sterben und Tod ihrer Patientinnen und Patienten. Der Geltungsbereich umfasst neben Menschen, bei denen der Sterbeprozess bereits eingesetzt hat, auch solche, bei denen eine wahrscheinlich tödlich verlaufende Krankheit diagnostiziert wurde, sowie Personen, die medizinische Hilfe zur Beendigung ihres Lebens wünschen. Ein Kapitel über ethische Grundsätze erläutert das Recht auf Selbstbestimmung, den sozialen Kontext der Selbstbestimmung, die Begriffe «Lebensqualität» sowie «Leiden» und "Leidenslinderung». Weitere Themen betreffen die Wichtigkeit der Klärung des Behandlungsziels sowie der Betreuung der Angehörigen. In den beiden folgenden Kapiteln werden Anleitungen zum Gespräch über Sterben und Tod und zum Umgang mit Sterbewünschen gegeben. Das Kapitel über Entscheidungsprozesse legt grossen Wert auf die gemeinsame Entscheidungsfindung und die Vorausplanung von Behandlung und Betreuung.

Bei den "Handlungen, die - möglicherweise oder sicher - den Eintritt des Todes beschleunigen» werden drei Kategorien unterschieden: 1. allgemein akzeptierte Handlungen, wie Unterlassung und Abbruch lebenserhaltender Massnahmen, Linderung von Schmerzen und anderen Symptomen sowie Sedierung; 2. kontrovers diskutierte Handlungen, dazu gehört die Suizidhilfe und das Unterstützen des freiwilligen Verzichts 
auf Nahrung und Flüssigkeit; 3. nicht zulässige Handlungen, die rechtlich verboten sind. Im Anhang der Richtlinien werden Behandlungsziele und Handlungen im Umgang mit Sterben und Tod definiert und ethische Überlegungen $\mathrm{zu}$ den Zielen der Medizin angeführt.

\section{Kontrovers diskutierte Handlungen}

Für die kontrovers diskutierten Handlungen formulieren die Richtlinien ethische Leitplanken. Für diese Handlungen gibt es - im Gegensatz zu den allgemein akzeptierten medizinischen Massnahmen - keine medizinische Indikation. Der Arzt muss entscheiden, ob Suizidhilfe mit seinem berufsethischen Selbstverständnis vereinbar ist und ob er aufgrund seiner Überzeugung im konkreten Fall zum Wohl des Patienten handeln kann. Eine im Auftrag der SAMW 2014 durchgeführte Studie zur Haltung der Ärzteschaft ${ }^{1}$ hat gezeigt, dass viele Ärzte Suizidhilfe zwar für vertretbar halten, doch nur eine Minderheit bereit ist, selbst bei einem Suizid Unterstützung zu leisten. Die Richtlinien betonen denn auch, dass Patienten keinen Anspruch auf Suizidhilfe haben und es jedem Arzt freisteht, diese Handlung für sich in Betracht zu ziehen oder nicht.

Angesichts des rechtlichen Rahmens und der unterschiedlichen Haltungen in der Ärzteschaft, bei anderen medizinischen Fachpersonen und in der Gesellschaft betonen die Richtlinien, dass sich das bestehende Spannungsfeld nicht auflösen lässt: Ethische Forderungen, die Selbstbestimmung zu fördern, stehen dem Willen gegenüber, das Leben zu schützen. Trotzdem muss es für die medizinischen Fachpersonen möglichst klare Leitplanken geben.

Die Formulierung objektiver medizinischer Kriterien für die Zulässigkeit der Suizidhilfe ist problematisch. Einerseits führt dies zu schwierigen Abgrenzungsfragen (was ist eine tödliche Krankheit?), andererseits könnten bei Vorliegen einer tödlichen Krankheit sowohl Patienten als auch Behandelnde unter Rechtfertigungsdruck geraten, wenn sie die Möglichkeit der Suizidhilfe nicht in Betracht ziehen wollen. Meist ist es auch nicht der medizinisch objektivierbare Zustand, der bei der Patientin zum selbstbestimmten Suizidwunsch führt, sondern das subjektiv erlebte unerträgliche Leiden. Dieses lässt sich jedoch nicht objektivieren. Um zu rechtfertigen, dass die Suizidhilfe überhaupt in den medizinischen Zuständigkeitsbereich fällt, müssen dem unerträglichen Leiden aber medizinisch fassbare Krankheitssymptome oder Funktionseinschränkungen zugrunde liegen. Für den Arzt muss das Leiden intersubjektiv nachvollziehbar sein.

\section{Regelung der Suizidhilfe}

Kapitel 6.2.1. der Richtlinien lautet wie folgt:

«Gemäss Art. 115 des Strafgesetzbuches ist die Beihilfe zum Suizid straflos, wenn sie ohne selbstsüchtige Beweggründe erfolgt. Dies gilt für alle Personen. Äussert ein Patient den Wunsch nach Suizidhilfe, ist dies ein Sterbewunsch und entsprechend sorgfältig abzuklären. Dabei soll der Patient auch motiviert werden, mit den Angehörigen über seinen Suizidwunsch zu sprechen.

Die Rolle des Arztes im Umgang mit Sterben und Tod besteht darin, Symptome zu lindern und den Patienten zu begleiten. Es gehört weder zu seinen Aufgaben, von sich aus Suizidhilfe anzubieten, noch ist er verpflichtet, diese zu leisten. Suizidhilfe ist keine medizinische Handlung, auf die Patienten einen Anspruch erheben könnten, sie ist jedoch eine rechtlich zulässige Tätigkeit. Sie kann vom Arzt geleistet werden, wenn er sich überzeugt hat, dass die untenstehenden Voraussetzungen erfüllt sind.

Zur Suizidhilfe zählen Handlungen, die in der Absicht erfolgen, einer urteilsfähigen Person die Durchführung des Suizids zu ermöglichen, insbesondere die Verschreibung oder Aushändigung eines Medikamentes zum Zweck der Selbsttötung. Die Verschreibung eines Medikaments zum Zweck der Selbsttötung muss innerhalb von 30 Tagen den zuständigen kantonalen Behörden gemeldet werden. Wie jeder Patient hat auch derjenige, der sich unter Beizug einer Suizidhilfeorganisation das Leben nehmen möchte, Anspruch auf Einsichtnahme in sein Dossier und eine Kopie desselben. Wenn der Patient es wünscht, kann der betreuende Arzt auch eine Prüfung der kognitiven Funktionen vornehmen und ggf. eine Bestätigung der Urteilsfähigkeit für allgemeine Entscheidungen des Alltags ausstellen. Eine solche Abklärung stellt keine Beteiligung am assistierten Suizid dar. Der Arzt kann auch eine Prüfung und ggf. Bestätigung der spezifischen Urteilsfähigkeit für einen assistierten Suizid (welche nicht aus der Urteilsfähigkeit für allgemeine Entscheidungen des Alltags hervorgeht) vornehmen. Eine solche Abklärung kann jedoch vom Patienten nicht eingefordert werden.

Bleibt nach sorgfältiger Information und Abklärung ein selbstbestimmter Wunsch nach Suizidhilfe bestehen, kann ein Arzt aufgrund eines persönlich verantworteten Entscheides Suizidhilfe leisten, wenn die folgenden fünf Voraussetzungen gegeben sind und er deren Erfüllung überprüft hat. Das Vorliegen der ersten beiden Voraussetzungen muss zusätzlich von einer unabhängigen Drittperson bestätigt werden; diese muss nicht zwingend ein Arzt sein: 
- Der Patient ist in Bezug auf den assistierten Suizid urteilsfähig. Der Arzt muss dokumentieren, dass er eine Urteilsunfähigkeit sorgfältig ausgeschlossen hat. Falls eine psychische Krankheit, eine Demenz oder ein anderer Zustand vorliegt, der häufig mit fehlender Urteilsfähigkeit verbunden ist, wurde die Urteilsfähigkeit durch einen entsprechenden Facharzt evaluiert.

- Der Wunsch ist wohlerwogen und ohne äusseren Druck entstanden sowie dauerhaft. Falls Hinweise auf ein problematisches Abhängigkeitsverhältnis bestehen, wurde dessen möglicher Einfluss auf den Suizidwunsch sorgfältig erwogen.

- Die Krankheitssymptome und/oder Funktionseinschränkungen des Patienten sind für diesen Ursache unerträglichen Leidens.

- Medizinisch indizierte therapeutische Optionen sowie andere Hilfs- und Unterstützungsangebote wurden gesucht und sind erfolglos geblieben oder werden vom diesbezüglich urteilsfähigen Patienten als unzumutbar abgelehnt.

- Der Wunsch des Patienten, in dieser unerträglichen Leidenssituation nicht mehr leben zu wollen, ist für den Arzt aufgrund der Vorgeschichte und wiederholter Gespräche nachvollziehbar und es ist für ihn vertretbar, in diesem konkreten Fall Suizidhilfe zu leisten.

Der letzte Akt der zum Tod führenden Handlung muss in jedem Fall durch den Patienten durchgeführt wer- den. Der Tod nach Suizidhilfe muss als aussergewöhnlicher Todesfall der zuständigen Behörde gemeldet werden. Im Vorfeld, während und nach der Suizidhilfe ist auf die Bedürfnisse der Angehörigen, aber auch des interprofessionellen Betreuungsteams und des Umfelds Rücksicht zu nehmen und die benötigte Unterstützung zu geben.»

\section{Hauptanliegen bleibt das Patientenwohl}

Die Richtlinien fallen in eine Zeit rascher Entwicklungen und heftiger Kontroversen darüber, was von Ärzten und anderen medizinischen Fachpersonen im Umgang mit Sterben und Tod erwartet werden soll. Sie streben eine Vermittlung zwischen unterschiedlichen Sichtweisen und Wertvorstellungen an und wirken darauf hin, dass die Selbstbestimmung aller Beteiligten - der Patientinnen, der Angehörigen und der medizinischen Fachpersonen - geachtet und geschützt wird. Das Hauptanliegen eines am Patientenwohl orientierten Umgangs mit Sterben und Tod darf weder zu einer Überforderung der Angehörigen noch zu einer Gefährdung des professionellen Selbstverständnisses der medizinischen Fachpersonen führen.

Die Richtlinien stehen online in vier Sprachen (D/E/F/I) zur Verfügung und können kostenlos gedruckt bestellt werden (D/F): samw.ch/richtlinien 